\title{
Hemodynamics analyses in treated and untreated carotid arteries of the same patient: a preliminary study based on three patient cases $^{1}$
}

\author{
Yuqian Mei ${ }^{\mathrm{a}}$, Matthias Müller-Eschner ${ }^{\mathrm{b}, \mathrm{c}}, \mathrm{Jie} \mathrm{Yi}^{\mathrm{a}}$, Zhaotong Zhang ${ }^{\mathrm{a}}$, Duanduan Chen ${ }^{\mathrm{a}, *}$, \\ Moritz Kronlage ${ }^{\mathrm{b}}$, Hendrik von Tengg-Kobligk ${ }^{\mathrm{b}, \mathrm{d}}$, Hans-Ulrich Kauczor ${ }^{\mathrm{b}}$, Dittmar Böckler \\ and Serdar Demirel ${ }^{\mathrm{e}}$ \\ ${ }^{a}$ Department of Biomedical Engineering, School of Life Science, Beijing Institute of Technology, \\ Beijing, China \\ ${ }^{b}$ Department of Diagnostic and Interventional Radiology, University Hospital Heidelberg, Heidelberg, \\ Germany \\ ${ }^{c}$ Department of Radiology, German Cancer Research Center (dkfz), Heidelberg, Germany \\ ${ }^{d}$ Institute of Diagnostic, Interventional and Pediatric Radiology, University Hospital Bern, Inselspital, \\ Bern, Switzerland \\ ${ }^{e}$ Department of Vascular and Endovascular Surgery, University Hospital Heidelberg, Heidelberg, \\ Germany
}

\begin{abstract}
Carotid atherosclerotic disease is highly related to cerebrovascular events. Carotid endarterectomy is the common operation method to treat this disease. In this study, hemodynamics analyses are performed on the carotid arteries in three patients, whose right carotid artery had been treated by carotid endarterectomy and the left carotid artery remained untreated. Flow and loading conditions are compared between these treated and untreated carotid arteries and evaluation of the operative results is discussed. Patient-specific models are reconstructed from MDCT data. Intraoperative ultrasound flow measurements are performed on the treated carotid arteries and the obtained data are used as the boundary conditions of the models and the validations of the computational results. Finite volume method is employed to solve the transport equations and the flow and loading conditions of the models are reported. The results indicate that: (i) in two of the three patients, the internal-to-external flow rate ratio in the untreated carotid artery is larger than that in the treated one, and the average overall flow split ratio by summing up the data of both the left and right carotid arteries is about 2.15; (ii) in the carotid bulb, high wall shear stress occurs at the bifurcation near the external carotid artery in all of the cases without hard plaques; (iii) the operated arteries present low time-averaged wall shear stress at the carotid bulb, especially for the treated arteries with patch technique, indicating the possibility of the recurrence of stenosis; (iv) high temporal gradient of wall shear stress ( $>35 \mathrm{~Pa} / \mathrm{s})$ is shown in the narrowing regions along the vessels; and (v) in the carotid arteries without serious stenosis, the maximum velocity magnitude during mid-diastole is $32 \sim 37 \%$ of that at systolic peak, however, in the carotid artery with $50 \%$ stenosis by hard plaques, this value is nearly doubled (64\%). The computational work quantifies flow and loading distributions in the
\end{abstract}

\footnotetext{
1 Yuqian Mei and Matthias Müller-Eschner are the first authors of this paper.

* Address for correspondence: Duanduan Chen, School of Life Science, Beijing Institute of Technology, Beijing, China. Tel.: +86 10 68912154; Fax: +86 10 68915956; E-mail: duanduan@bit.edu.cn.
} 
treated and untreated carotid arteries of the same patient, contributing to evaluation of the operative results and indicating the recurrent sites of potential atheromatous plaques.

Keywords: Carotid artery, computational fluid dynamics, hemodynamics, carotid endarterectomy

\section{Introduction}

Atherosclerotic changes, typically occurring near the bifurcation of the common carotid artery, are an important cause of ischemic cerebrovascular events by either representing a source of embolism or locally occluding the internal carotid artery as the "main channel of blood supply" to the brain [1]. Carotid endarterectomy (CEA) is extensively used to treat carotid artery stenosis. Inducement of restenosis after CEA treatment remains a problem that is not yet fully understood [2]. Among various factors, which are involved in the establishment of restenosis, hemodynamic conditions play an important role $[3,4]$. In recent years, computational fluid dynamics have become a useful method in studying the pathology of carotid artery disease and evaluating the post-treatment effects [5-7]. Several mechanical parameters such as wall shear stress (WSS) have been reported to present close relationships to the establishment of plaque and restenosis $[4,8,9]$. Some of the studies correlated preand post-operative hemodynamic parameters $[10,11]$; however, very few of them compared the operated vessel to the contralateral, untreated carotid artery [12]. In fact, the left and right carotid arteries together with the vertebral arteries function systematically to supply blood to the brain and other facial organs. Since the morphology of treated and untreated carotid arteries of the same subject is not necessarily similar, comparing the hemodynamics of them may contribute to improving our understanding of the blood transportation to the brain. Moreover, this may help evaluate the postendarterectomy results, by not only comparing to the pre-operative stenosis conditions, but also comparing to its untreated counterpart and studying the balance of blood transportation between them.

In this study, we establish computational models based on computed tomography scans of three patients, each of whom had been treated by carotid-endarterectomy on one side while the contralateral side remained untreated. Ultrasound flow measurements are used to provide inflow conditions of the models as well as validations of the results. Detailed flow and loading information of both the left and right carotid arteries are reported by solving the 3D unsteady conservation equations of mass and momentum. Velocity, pressure, wall shear stress and its deductive variables are investigated. Finally, the evaluations of post-operative hemodynamics are discussed.

\section{Methods}

Institutional review board approval was obtained for this retrospective analysis. All three patients received a multidetector computed tomography scan (MDCT) one day after surgery. Patient I and II (PI and PII) were scanned with a Somatom Definition (Siemens Medical Systems, Erlangen, Germany). Scan and reconstruction parameters were as follows: $120 \mathrm{kV}, 120$ ref. mAs, reconstructed slice thickness $1 \mathrm{~mm}$, reconstruction increment $0.7 \mathrm{~mm}$, pixel spacing $0.5 \times 0.5 \mathrm{~mm}$ and $90 \mathrm{ml}$ contrast medium (iomeprol with 350mg iodine per ml, Imeron 350, Bracco Diagnostics, Princeton, NJ, USA) with $50 \mathrm{ml}$ saline chaser. Patient III (PIII) was scanned with a Definition (Siemens Medical Systems, Erlangen, Germany). Scan and reconstruction parameters were as follows: $120 \mathrm{kV}, 120 \mathrm{mAs}$, reconstructed slice thickness $0.75 \mathrm{~mm}$, reconstruction increment $0.7 \mathrm{~mm}$, pixel spacing $0.4 \times 0.4 \mathrm{~mm}$ and 
$90 \mathrm{ml}$ contrast medium (iomeprol with $400 \mathrm{mg}$ iodine per $\mathrm{ml}$, Imeron 400, Bracco Diagnostics, Princeton, NJ, USA) with $50 \mathrm{ml}$ saline chaser. The MDCT datasets of the right carotid arteries of PI and PIII have been used in our previous study [13]. In the current study, these two datasets were newly segmented and simulations were performed based on the newly reconstructed models. Intraoperative flow measurements within the internal carotid (ICA) and common carotid artery (CCA) were assessed by intraoperative ultrasound (MediStim $\subset$, VeriQ $\mathbb{C}$, Deisenhof, Germany). The flow information by this method provides boundary conditions of the computational work and is used to validate the results.

The segmentation and surface reconstruction of the carotid arteries were accomplished by a semiautomatic threshold-based segmentation tool (Amira 5.4, Visage Imaging Inc., USA). Figures 1a-1c displays the sagittal computed tomography reconstructions of the left and right carotid arteries for PIPIII, respectively. And Figures 1d-1f displays the models of the left and right carotid arteries of PI, PII and PIII, respectively. The reconstructed geometries were meshed by ICEM CFD (ANSYS 14.0 Inc., USA) with tetrahedral elements in the core region and prismatic cells in the boundary layer near the vessel wall (8 layers of prismatic cells define the near-wall region). The left external carotid artery (ECA) of PIII has been artificially extended for the simulation in order to reduce the pressure difference at the outlets (Figures $2 \mathrm{f}-2 \mathrm{~L}$ ). The baseline grid resolution of the left and right carotid arteries of PI possess 1,389,768 and 1,796,884 cells, respectively; while those are 1,137,429 and $1,321,723$ cells for PII, and 1,496,672 and 1,142,203 cells for PIII.

Numerical simulation was performed by a Finite Volume solver, CFD-ACE (ESI-Group, France) by solving the transport equations - the Naviér-Stokes equations. Blood was treated as an incompressible and Newtonian fluid with a density of $1050 \mathrm{~kg} / \mathrm{m}^{3}$ and a dynamic viscosity of $0.0035 \mathrm{~Pa} \cdot \mathrm{s}$ [14]. The average Reynolds number in these models varies between 649 to 793; thus, a laminar model of the flow was applied during the computation [15]. A second order accurate discretization (central differences) was used to solve the flow velocity. Algebraic MultiGrid acceleration was employed and the SIMPLEC-type pressure correction was used for pressure-velocity coupling.

Pulsatile flow information was derived from the waveforms described in older human subjects [16]. Parabolic velocity profile was applied at the inlet of CCA [17], and the maximum velocity at the inlet along the centreline of CCA was calculated based on the measured volumetric flow rate. Flow measurements have only been conducted for the operated carotid arteries and they are $379 \mathrm{ml} / \mathrm{min}$, $362 \mathrm{ml} / \mathrm{min}$, and $360 \mathrm{ml} / \mathrm{min}$ in the CCA, for PI-PIII respectively. Previous studies indicate the flow entering the left and right carotid arteries are nearly the same [18]. Therefore, in each case, the same inlet flow rate condition was applied on both the left and right carotid arteries and was based on the measured data of the operated carotid arteries. For each cardiac cycle, 69 time steps were involved in the baseline models, indicating a time period of $0.011594 \mathrm{~s}$ for each time step. No-slip boundary conditions were imposed at the vessel walls and zero-pressure boundary conditions were applied at the outlets of ICA and ECA.

To confirm the insensitivity of the results to the spatial resolution and time, we conducted a grid independence analysis and a time step sensitivity test on the left carotid artery model of PI as a representative. Apart from the base discretization $(1,389,768$ cells) and the base time step $(0.011594 \mathrm{~s}$, 69 steps per cycle), the solutions on a finer grid (7,207,174 cells) with a finer temporal discretization of $0.0058 \mathrm{~s}$ (137 steps per cycle) has been investigated. To compare the results, the flow pattern at the systolic peak is studied and a point at the centre of the carotid bulb has been selected to compare the velocity magnitudes between the two models. The results show exactly the same trend of flow pattern of the two resolutions and the maximum discrepancies of the velocity magnitude between the two models at the same studying point in the carotid bulb at systolic peak is $1.84 \%$. This confirms that the grid and time resolution of the base model in PI is adequate for the current study. Since the models of 


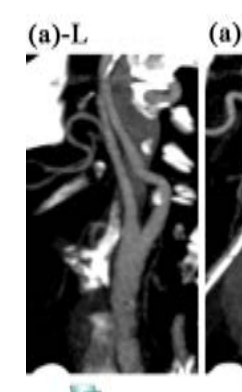

(d)-
(a)-R

(b)-L

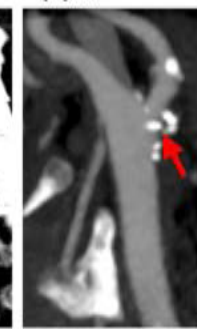

(d)-R

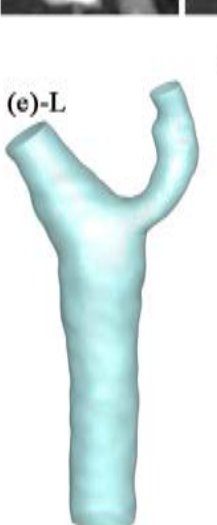

(b) $=\mathrm{R}$

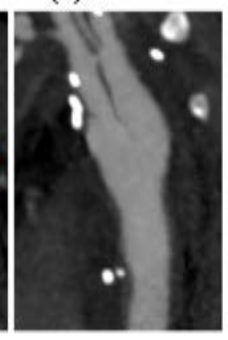

(e)-R

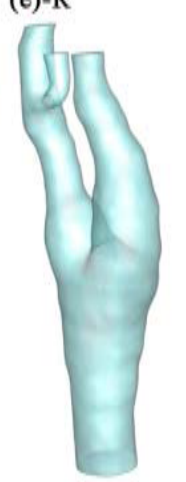

(c)-L

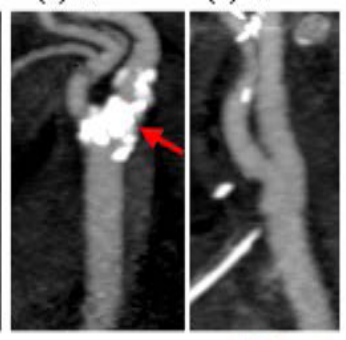

(f)-L

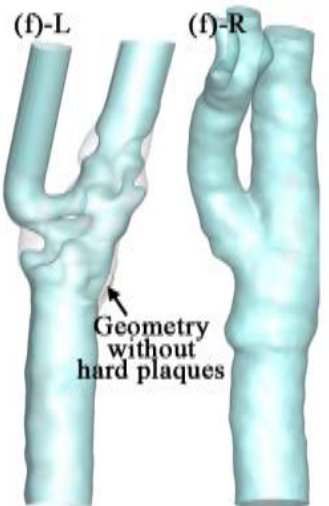

Fig. 1. (a)-(c) and (d)-(f) display the sagittal image of datasets and reconstructed models for PI-PIII, respectively. Red arrows indicate soft plaques in left carotid artery of PII and hard plaques in left carotid artery of PIII. Grey transparent structure in (f)-L (pointed by the black arrow) simulates geometry by artificially removing hard plaques.

PII and PIII present similar grid resolutions and are solving similar flow problems, we believe the baseline grid and time discretizations are adequate. The simulation is carried out for 5 cardiac cycles to achieve a stable solution and the results of the final cycle are presented below.

\section{Results and discussion}

All three patients underwent CEA of the right carotid artery; while the left carotid artery remained untreated. Figures 1a-c displays the sagittal computed tomography reconstructions of the left and right carotid arteries for PI-PIII, respectively. No stenosis is detected in the post-operative carotid arteries in all patients (Figures 1a-R, b-R and c-R); while for the untreated ones, the situation varies: in PI, no obvious/serious plaque or stenosis is detected (Figures 1a-L); in PII, 20-30\% narrowing caused by soft plaques presents at the bifurcation (Figures 1b-L); however, in PIII, 50\% stenosis caused by hard plaques is present at the bifurcation (Figures 1c-L). Investigations of the flow and loading patterns of left and right carotid arteries for PI-PIII will be discussed in the following.

\subsection{Flow analysis and validations}

Figures 2a-c displays the flow patterns of the left and right carotid arteries for PI-PIII at systolic peak, respectively, by drawing the streamlines. The operated and untreated carotid arteries in PI and PII and the operated artery in PIII present fairly organized flow throughout the vessel. Relatively high velocities $(0.46-0.78 \mathrm{~m} / \mathrm{s})$ are shown along the centreline of CCA and ICA in these cases, indicating 

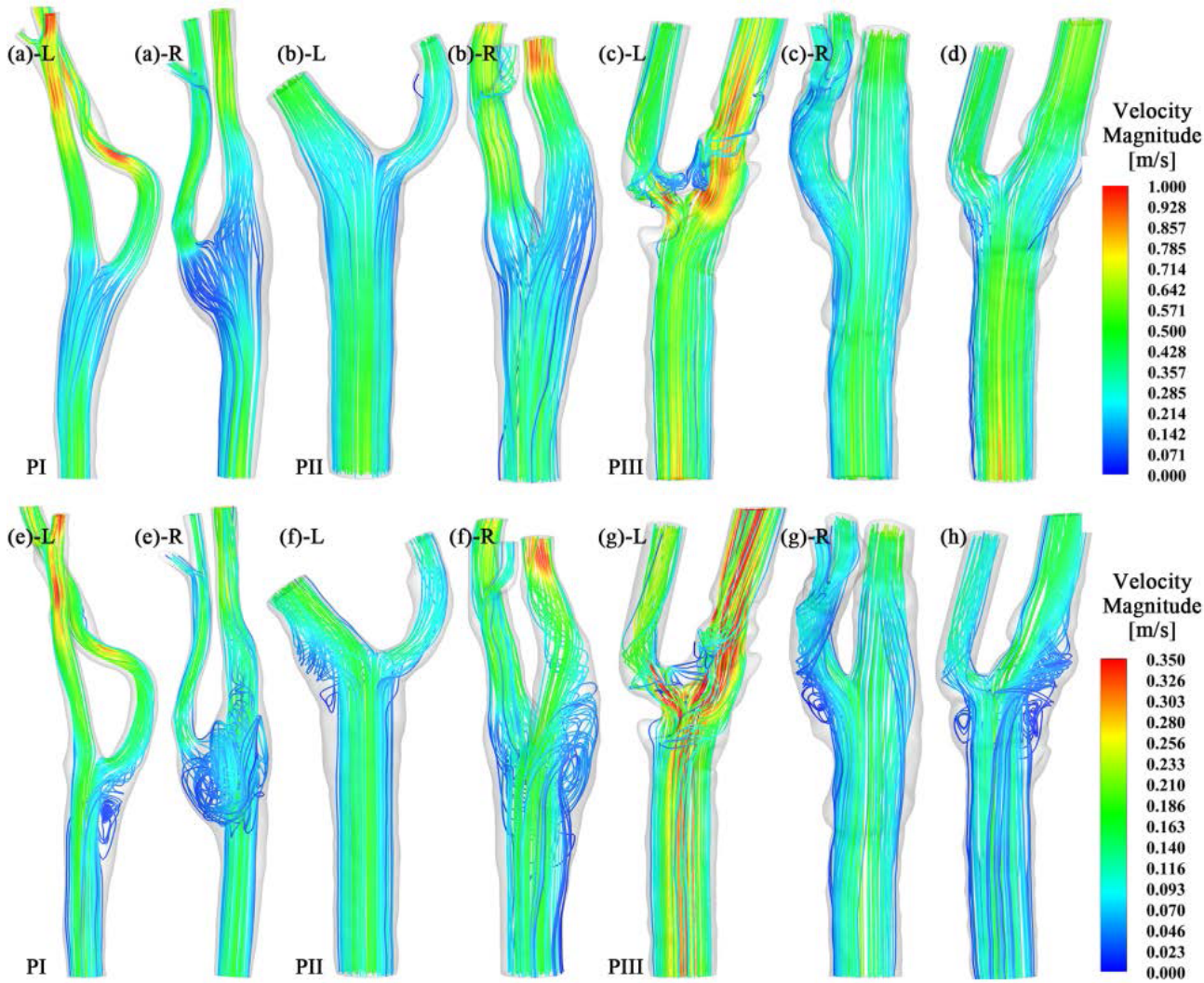

Fig. 2. Flow patterns of the models. (a)-(c) and (f)-(g) display flow patterns of left and right carotid arteries for PI-PIII at systolic peak and during mid-diastole, respectively. (d) and (h) show flow patterns of left carotid artery of PIII based on geometry without hard plaques at systolic peak and mid-diastole, respectively.

strong blood transports (with high flow rates) in these vessels. However, in the untreated carotid artery of PIII, the flow in the bifurcation is greatly disturbed by hard plaques: high velocities up to $1.08 \mathrm{~m} / \mathrm{s}$ occur in the bifurcation and vortical features can be observed. We conducted a further flow test on the untreated artery in PIII by artificially removing all the plaques. Figure $2 \mathrm{~d}$ shows, without those plaques, the flow at systolic peak returns to the organized pattern as observed in other cases and the maximum velocity decreases to $0.63 \mathrm{~m} / \mathrm{s}$, indicating the hemodynamic improvements that might be achieved by surgery. Moreover, the carotid bulbs in the treated side of PI and PII are enlarged by the patch technique during CEA and thus induce low flow velocities in these regions (Figures 2a-R and 2b-R).

Figure 2e-g displays the flow patterns of the left and right carotid arteries for PI-PIII at mid-diastole, respectively, by drawing the streamlines. The flow shows chaotic features in the carotid bulb, especially for the enlarged ones in the treated side of PI and PII (Figures 2e-R and 2f-R); while in the untreated side of PII (Figure 2f-L) and operated side of PIII (Figure 2g-R), the flow pattern is more ordered which may possibly be caused by the evenly distributed shape of the carotid bulb in these two cases. Comparing the results to those at systolic peak, the maximum velocity magnitude at middiastole is about $32-37 \%$ of that at systolic peak; however, in the untreated side of PIII (Figure 2g-L), this value rises up to $64 \%$. Figure $2 \mathrm{~h}$ shows the results of the untreated side of PIII by artificially removing the plaques. The maximum velocity magnitude of this case decreases to $32 \%$ of that at 
systolic peak, similar to the results obtained by other cases. This indicates, for the carotid arteries without obvious or serious stenosis, the velocity magnitude during mid-diastole could be reduced to approximately $35 \%$ of that at the systolic peak; however, for the carotid arteries with serious stenosis, this value could be largely increased. The results also imply that the $20-30 \%$ narrowing caused by soft plaques in the untreated side of PII has little effects on the hemodynamics of the carotid artery.

The intraoperative measured mean flow rates near the origin of ICA for the right-side of PI and PIII are $239 \mathrm{ml} / \mathrm{min}$ and $257 \mathrm{ml} / \mathrm{min}$, respectively, and the mean flow rates at the CCA are $379 \mathrm{ml} / \mathrm{min}$ and $360 \mathrm{ml} / \mathrm{min}$. To compare our results with the measured data, instantaneous flow rates were recorded at the proximal site of the ICA and ECA. The calculated mean flow rates at the ICA for the right-side of PI and PIII were $244.79 \mathrm{ml} / \mathrm{min}$ and $245.68 \mathrm{ml} / \mathrm{min}$, respectively, with discrepancies of $2.4 \%$ and $4.4 \%$ to the data acquired by intraoperative ultrasound. For the PII, the instantaneous maximum velocity magnitude is recorded in the ICA near the bifurcation which is $0.53 \mathrm{~m} / \mathrm{s}$ and the calculated maximum velocity magnitude is $0.54 \mathrm{~m} / \mathrm{s}$, showing the discrepancy of $1.9 \%$. The computational flow results can therefore be validated and further investigations regarding the loading distributions can be established.

\subsection{Volume flow rate}

Volume flow rate of ICA and ECA in each case over a cardiac cycle has been recorded at the proximal position. The ratio of the mean flow rate between ICA and ECA is calculated for each case: 1.62 for the left carotid artery of PI, 1.82 for the right carotid artery of PI, 2.97 for the left carotid artery of PII, 1.91 for the right carotid artery of PII, 4.37 for the left carotid artery of PIII, 2.16 for the right carotid artery of PIII, and if we artificially remove the plaques in the left carotid artery of PIII, its internal-to-external ratio of mean flow rate changes to 2.56. In PII and PIII (with or without the plaques), internal-to-external flow rate ratio in the left (untreated) carotid artery is larger than that of the right (treated) one, which is consistent to previous experimental reports [18]. The internal-toexternal ratio is proposed to be $1.46 \sim 3[16,19,20]$. In PI, PII, the operated side of PIII, and the model of the untreated side of PIII without plaques, the flow split is close to this range; while the untreated side of PIII (with plaques) is beyond this range.

To further investigate the flow rate, we also calculated the overall internal-to-external flow rate ratio by using the sum of the flow rate for both the right and the left carotid arteries of the same patient. The ratios for PI-PIII are 1.72, 2.37, and 3.23 (with plaque)/2.35 (without plaque), respectively. Comparing to the experimentally reported range (1.46-3), the overall flow condition of the carotid arteries in PI and PII is within the normal range, while that in PIII is beyond normal range. By artificially removing the plaques, overall flow split between internal and external carotid arteries in PIII reduces to 2.35, returning back to the normal range of the flow split ratio. The inherent anatomical difference of the common carotid arteries, arising from the aorta and the brachiocephalic truncus, may imply their variations in flow division. The current work confirms that effective treatments of carotid artery by removing the plaques can maintain the internal-to-external flow division within the normal range. However, this preliminary study only focuses on three patient cases; more reliable results should be achieved when multiple datasets are available.

\subsection{Loading distributions}

Figure 3a-c displays the pressure distributions of the left and right carotid arteries for PI-PIII at systolic peak, respectively. Consistent to the flow patterns, relatively high pressure is shown at the 


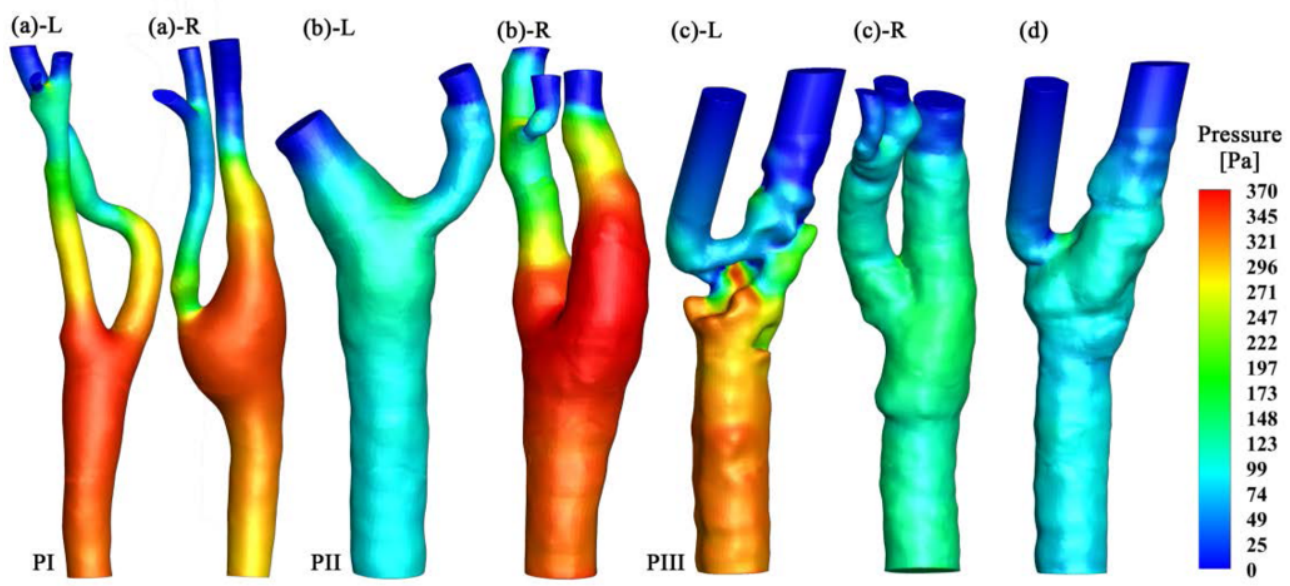

Fig. 3. Pressure distributions of the models. (a)-(c) display pressure distributions of left and right carotid arteries for PI-PIII at systolic peak, respectively. (d) shows pressure distribution of left carotid artery of PIII based on geometry without hard plaques at systolic peak.

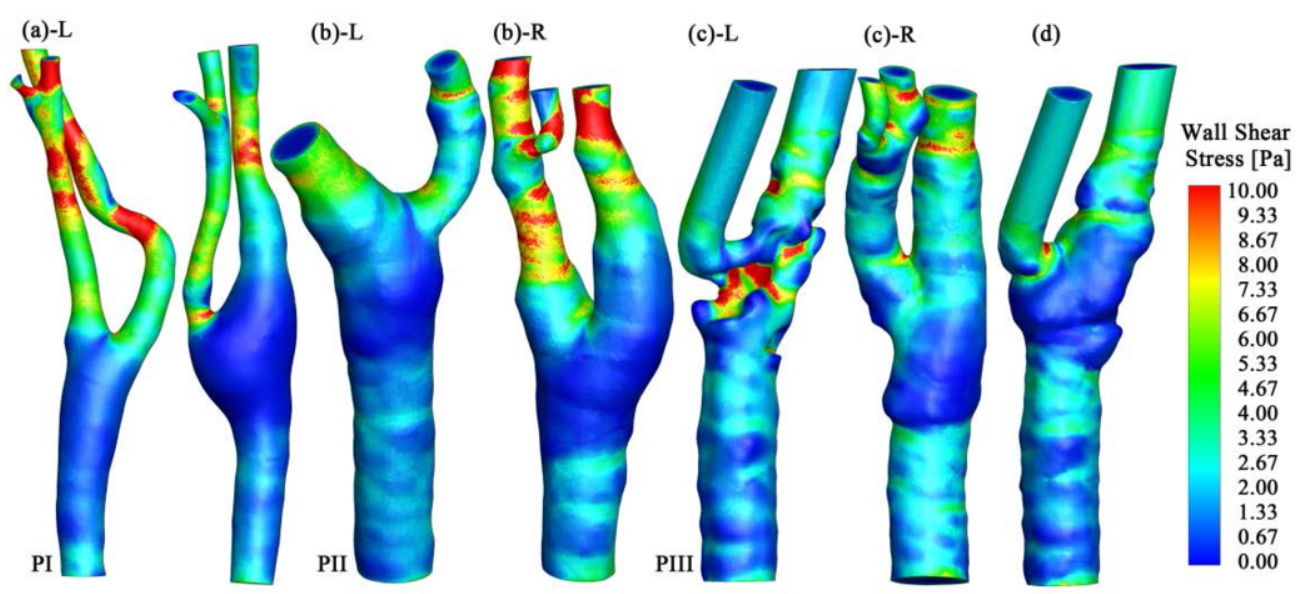

Fig. 4. WSS distributions of the models. (a)-(c) display WSS distributions of left and right carotid arteries PI-PIII at systolic peak, respectively. (d) shows WSS distribution of left carotid artery of PIII based on geometry without hard plaques at systolic peak.

carotid-bulb in the left and right carotid artery of PI, the operated side of PII, and the untreated side of PIII, where vortical flow with low velocities presents. The carotid bulb in the treated side of PI and PII is enlarged by the patch technique and high pressure (326-413 $\mathrm{Pa}$ ) presents at these regions. On the other hand, the physiological shape of the vessel endarterectomised without patch angioplasty (treated side of PIII) is well maintained and the pressure decreases gradually from the inlet of the model to the outlets; the highest pressure at this carotid bulb is $178 \mathrm{~Pa}$. Figure $3 \mathrm{~d}$ presents pressure distributions of the untreated side (left carotid artery) of PIII by artificially removing the plaques. It shows pressure in the carotid bulb can be reduced greatly (from $350 \mathrm{~Pa}$ to $192 \mathrm{~Pa}$ ) by removing the plaques, indicating the obstructive effects produced by the stenosis.

Figure 4a-c displays the WSS distributions of the left and right carotid artery for PI-PIII at systolic peak, respectively; and Figure 4d shows the WSS distribution of the left carotid artery of PIII after 
removing the plaques. In PI, PII and the case of PIII without plaques, the carotid bulb is experienced low WSS (0-2 Pa); while high WSS (9-14 Pa) presents in the interior region of the ECA near the bifurcation. For the untreated side of PIII, high WSS up to $30 \mathrm{~Pa}$ occurs near the stenosis, where the flow presents most chaotic features. WSS is reported to be related to endothelial cell function and is an important factor in the progression of atherosclerosis [21, 22]. High WSS over $30 \mathrm{~Pa}$ may induce desquamation of endothelial cells, platelet aggregation, intimal injury or rupture of vulnerable plaques $[23,24]$. The WSS distributions in all of the cases are below 30Pa, except for the stenosis at the carotid bulb and the proximal ICA of PIII.

In order to further investigate the loading pattern, two deductive variables of WSS are studied: the time-averaged WSS (TAWSS) and the maximum temporal gradient of WSS (TGWSS). The former is defined as the time-averaged magnitude of the WSS vector; the latter is calculated as $\operatorname{TGWSS}_{i}=\max \left(\partial \mid \boldsymbol{\tau} \boldsymbol{\tau}_{i}\right.$ $/ \partial t$ ), where $i$ is the spatial point. Figures 5 and 6 display the TAWSS and TGWSS distributions, respectively. Subfigures a-c display the results of left and right carotid artery for PI-PIII, respectively;
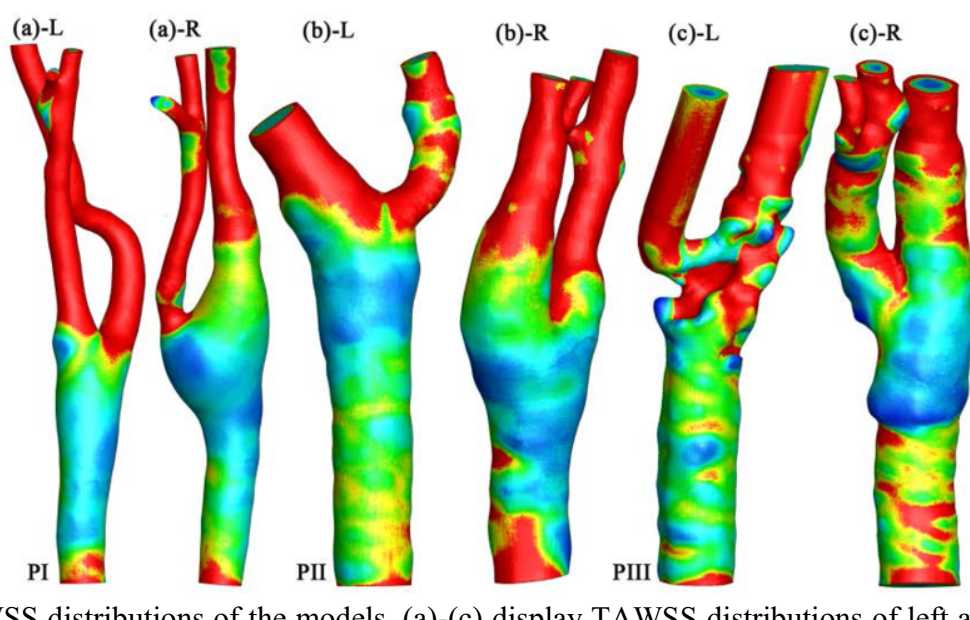

(d)

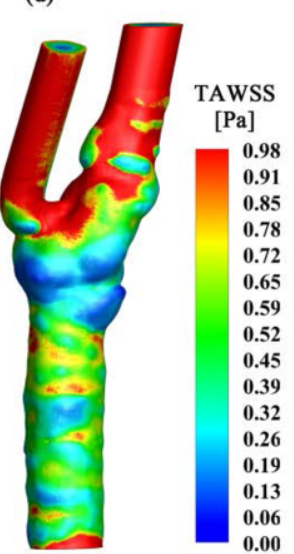

Fig. 5. TAWSS distributions of the models. (a)-(c) display TAWSS distributions of left and right carotid arteries for PI-PIII, respectively. (d) shows TAWSS distribution of left carotid artery of PIII based on geometry without hard plaques.

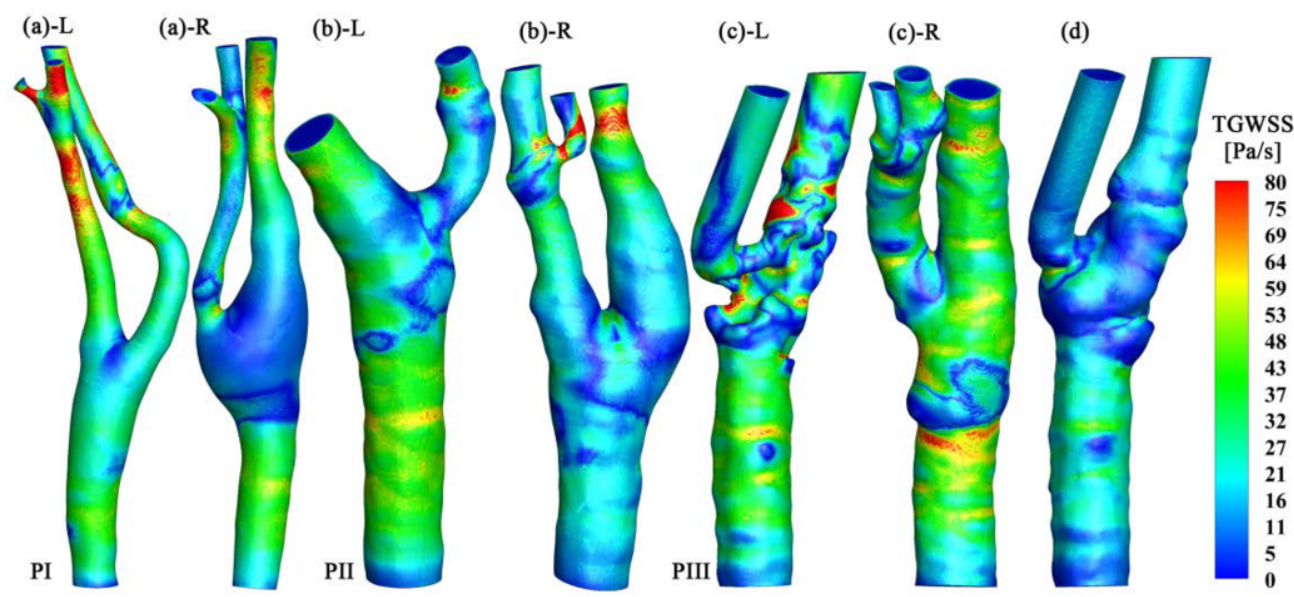

Fig. 6. TGWSS distributions of the models. (a)-(c) display TGWSS distributions of left and right carotid arteries for PI-PIII, respectively. (d) shows TGWSS distribution of left carotid artery of PIII based on geometry without hard plaques. 
and subfigure $d$ shows the results of the left carotid artery of PIII after removing the plaques. The TAWSS of the six cases ranges $0.03-9.74 \mathrm{~Pa}$. The colour map of Figure 5 has been restricted to $0-1 \mathrm{~Pa}$, in order to clearly display the low TAWSS regions. Low TAWSS $(<0.5 \mathrm{~Pa})$ occurs at the carotid bulb in all cases except for the stenosis in PIII. After removing the plaques, the carotid bulb in PIII also shows TAWSS smaller than $0.5 \mathrm{~Pa}$ (Figure 5d). In the right carotid artery of PI and PII, low TAWSS also displays in the CCA at the region close to the carotid bulb, which is possibly related to the CEA with patch angioplasty applied on these arteries; while, in other cases, this situation is not obvious. The low TAWSS region in the treated carotid arteries of PI and PII is much larger than that in the treated carotid artery of PIII. Low TAWSS less than $0.5 \mathrm{~Pa}$ may stimulate atherogenic phenotype, indicating a higher possibility of the recurrent atheromatous plaques at the carotid bulb and the region of CCA close to the carotid bulb in the treated carotid artery of PI and PII.

As shown in Figure 6, high TGWSS ( $>60 \mathrm{~Pa} / \mathrm{s})$ occurs at the narrowing regions along the vessel. It is reported that high values of TGWSS over $35.5 \mathrm{~Pa} / \mathrm{s}$ are related to the expression of atherogenesisrelated genes in endothelial cells $[25,26]$. This may indicate the vulnerability of the narrowing regions that may potentially suffer from atherosclerosis. Focusing on the carotid bulb alone, the operated carotid artery of PIII shows a small region with TGWSS higher than $35 \mathrm{~Pa} / \mathrm{s}$; while relatively low TGWSS less than $25 \mathrm{~Pa} / \mathrm{s}$ is shown in the operated carotid artery of PI and PII. In the carotid bulbs of the untreated vessels in PI and PII, no high TGWSS is observed; while, in the untreated vessel of PIII, high TGWSS is shown which is induced by the stenosis. By artificially removing these hard plaques, Figure $6 \mathrm{~d}$ shows no high TGWSS in the carotid bulb for this case.

\section{Limitations}

First, only three patient cases are studied in the current work. Future studies involving multiple datasets should be pursued to achieve more characteristic and reliable results. Second, due to the lack of experimental measured data, the outflow pressure in the ECA and ICA for all of the cases is assumed as zero; although acceptable flow validation is presented by comparing the computed and measured flow rate in the current work. Finally, the compliance of the carotid artery wall is not considered in this study at this stage.

\section{Conclusions}

We established computational models of the left and right carotid arteries of three patients by reconstructing the geometries from CTA data. Flow studies indicate that: (i) in two of the three patients, the internal-to-external flow rate ratio in the untreated carotid artery is larger than that in the treated one, and the average overall flow split ratio in PI, PII and the model of PIII without plaques is 2.15 , which is within the experimentally reported range; while the overall flow split in the model of PIII with stenosis is 3.23 , beyond the normal range; (ii) in the carotid arteries without serious stenosis, the maximum velocity magnitude during mid-diastole is $32-37 \%$ of that at systolic peak, however, in the carotid artery with hard plaque, this value is nearly doubled (64\% of the maximum velocity magnitude at systolic peak). Loading distribution studies indicate that: (i) in the carotid bulb, high WSS occurs at the bifurcation (near the ECA) in all of the cases, except for the stenosis case of PIII; (ii) in the operated arteries, the enlarged carotid bulbs in the right carotid artery of PI and PII present higher pressure and larger low TAWSS region than that in the right carotid artery of PIII; and (iii) high 
TGWSS is shown in the narrowing regions along these vessels, indicating the vulnerability of these regions. In summary, patient-specific models of carotid arteries can provide detailed flow and loading information of the blood transport. They can indicate possible recurrent sites of atheromatous plaques. Moreover, they can compare the hemodynamic parameters of untreated and operated carotid arteries and discuss overall blood supply by both the left and right carotid arteries. The current study investigates three patient cases only. More characteristic results, especially for the external-to-internal carotid artery flow split studies, can be achieved by involving more datasets, the next step of this research.

\section{Acknowledgment}

This work is supported by the National Natural Science Foundation of China (31200704 and 81471752). Matthias Müller-Eschner and Hendrik von Tengg-Kobligk received support from the German Research Foundation (DFG) within project R03, SFB/TRR 125 "Cognition-Guided Surgery".

\section{References}

[1] J.R. Crouse, et al., Risk factors for extracranial carotid artery atherosclerosis, Stroke 18 (1987), 990-996.

[2] C.N. Antonopoulos, et al., Eversion versus conventional carotid endarterectomy: A meta-analysis of randomised and non-randomised studies, European Journal of Vascular and Endovascular Surgery 42 (2011), 751-765.

[3] M. Silvestrini, et al., Cerebral hemodynamics and cognitive performance in patients with asymptomatic carotid stenosis, Neurology 72 (2009), 1062-1068.

[4] M. Markl, et al., In vivo wall shear stress distribution in the carotid artery: Effect of bifurcation geometry, internal carotid artery stenosis, and recanalization therapy, Circ Cardiovasc Imaging 3 (2010), 647-655.

[5] D. Birchall, et al., Analysis of haemodynamic disturbance in the atherosclerotic carotid artery using computational fluid dynamics, European Radiology 16 (2006), 1074-1083.

[6] I. Marshall, et al., MRI and CFD studies of pulsatile flow in healthy and stenosed carotid bifurcation models, Journal of Biomechanics 37 (2004), 679-687.

[7] D. Martin, et al., Analysis of haemodynamic factors involved in carotid atherosclerosis using computational fluid dynamics, British Journal of Radiology 82 (2009), S33-38.

[8] C. Yang, et al., Impact of flow rates in a cardiac cycle on correlations between advanced human carotid plaque progression and mechanical flow shear stress and plaque wall stress, Biomedical Engineering Online 10 (2011), 61.

[9] D. Tang, et al., Correlations between carotid plaque progression and mechanical stresses change sign over time: A patient follow up study using MRI and 3D FSI models, Biomedical Engineering Online 12 (2013), 105.

[10] H. Hayase, et al., Computational fluid dynamics of carotid arteries after carotid endarterectomy or carotid artery stenting based on postoperative patient-specific computed tomography angiography and ultrasound flow data, Neurosurgery 68 (2011), 1096-1101.

[11] S. Hyun, C. Kleinstreuer and J.P. Archie, Computational particle-hemodynamics analysis and geometric reconstruction after carotid endarterectomy, Computers in Biology and Medicine 31 (2001), 365-384.

[12] A.K. Wakhloo, et al., Hemodynamics of carotid artery atherosclerotic occlusive disease, Journal of Vascular and Interventional Radiology 15 (2004), S111-121.

[13] S. Demirel, et al., Comparison of morphological and rheological conditions between conventional and eversion carotid endarterectomy using computational fluid dynamics - a pilot study, Vascular (2014), doi: 10.1177/1708538114552836, in press.

[14] H. Hayase, et al., Computational fluid dynamics of carotid arteries after carotid endarterectomy or carotid artery stenting based on postoperative patient-specific computed tomography angiography and ultrasound flow data, Neurosurgery 68 (2011), 1069-1101.

[15] H.F. Younis, H.F., et al., Hemodynamics and wall mechanics in human carotid bifurcation and its consequences for atherogenesis: Investigation of inter-individual variation, Biomechanics and Modeling in Mechanobiology 3 (2004), 1732. 
[16] Y. Hoi, et al., Characterization of volumetric flow rate waveforms at the carotid bifurcations of older adults, Physiological Measurement 31 (2010), 291-302.

[17] I.C. Campbell, et al., Effect of inlet velocity profiles on patient-specific computational fluid dynamics simulations of the carotid bifurcation, Journal of Biomechanical Engineering 134 (2012), 051001.

[18] P. Bendel, et al., Blood flow in the carotid arteries: Quantification by using phase-sensitive MR imaging, American Journal of Roentgenology 152 (1989), 1307-1310.

[19] D.N. Ku and D.P. Giddens, Pulsatile flow in a model carotid bifurcation, Arteriosclerosis, Thrombosis, and Vascular Biology 3 (1983), 31-39.

[20] J.S. Milner, J.A. Moore, B.K. Rutt and D.A. Steinman, Hemodynamics of human carotid artery bifurcations: Computational studies with models reconstructed from magnetic resonance imaging of normal subjects, Journal of Vascular Surgery 27 (1998), 143-156.

[21] A.M. Malek, S.L. Alper and S. Izumo, Hemodynamic shear stress and its role in atherosclerosis, Journal of American Medical Association 282 (1999), 2035-2042.

[22] A.M. Shaaban and A.J. Duerinckx, Wall shear stress and early atherosclerosis: A review, American Journal of Roentgenology 174 (2000), 1657-1666.

[23] C.M. Schirmer and A.M. Malek, Prediction of complex flow patterns in intracranial atherosclerotic disease using computational fluid dynamics, Neurosurgery 61 (2007), 842-851.

[24] C.J. Slager, et al., The role of shear stress in the generation of rupture-prone vulnerable plaques, Nature Clinical Practice Cardiovascular Medicine 2 (2005), 401-407.

[25] C.J. Slager, et al., The role of shear stress in the generation of rupture-prone vulnerable plaques, Nature Clinical Practice Cardiovascular Medicine 2 (2005), 401-407.

[26] X. Bao, C. Lu and J.A. Frangos, Temporal gradient in shear but not steady shear stress induces PDGF-A and MCP-1 expression in endothelial cells: Role of NO, NF kappa B, and egr-1, Arteriosclerosis, Thrombosis, and Vascular Biology 19 (1999), 996-1003. 